\title{
Characterization and Optical Compensation of LP01 and LP11 Intra-Modal Nonlinearity in Few-Mode Fibers
}

\section{Ros, Francesco Da; Kaminski, Pawel M.; Rademacher, Georg; Puttnam, Benjamin J.; Luis, Ruben S.; Klaus, Werner; Furukawa, Hideaki; Maruyama, Ryu; Aikawa, Kazuhiko; Morioka, Toshio}

Total number of authors:

13

Published in:

Proceedings of 2020 Optical Fiber Communications Conference and Exhibition

Link to article, DOI:

10.1364/OFC.2020.Th1H.4

Publication date:

2020

Document Version

Peer reviewed version

Link back to DTU Orbit

Citation (APA):

Ros, F. D., Kaminski, P. M., Rademacher, G., Puttnam, B. J., Luis, R. S., Klaus, W., Furukawa, H., Maruyama, R., Aikawa, K., Morioka, T., Oxenløwe, L. K., Wada, N., \& Galili, M. (2020). Characterization and Optical Compensation of LP and LP Intra-Modal Nonlinearity in Few-Mode Fibers. In Proceedings of 2020 Optical Fiber Communicatiohs Conference and Exhibition [9083234] IEEE. https://doi.org/10.1364/OFC.2020.Th1 H.4

\section{General rights}

Copyright and moral rights for the publications made accessible in the public portal are retained by the authors and/or other copyright owners and it is a condition of accessing publications that users recognise and abide by the legal requirements associated with these rights.

- Users may download and print one copy of any publication from the public portal for the purpose of private study or research.

- You may not further distribute the material or use it for any profit-making activity or commercial gain

- You may freely distribute the URL identifying the publication in the public portal 


\title{
Characterization and Optical Compensation of $\mathbf{L P}_{01}$ and LP$_{11}$ Intra-modal Nonlinearity in Few-Mode Fibers
}

\author{
Francesco Da Ros ${ }^{1, *}$, Pawel M. Kaminski ${ }^{1}$, Georg Rademacher ${ }^{2, \dagger}$, Benjamin J. Puttnam ${ }^{2}$, \\ Ruben S. Luis ${ }^{2}$, Werner Klaus ${ }^{2}$, Hideaki Furukawa ${ }^{2}$, Ryu Maruyama ${ }^{3}$, Kazuhiko Aikawa ${ }^{3}$, \\ Toshio Morioka ${ }^{1}$, Leif K. Oxenløwe ${ }^{1}$, Naoya Wada ${ }^{2}$, and Michael Galili ${ }^{1}$ \\ ${ }^{1}$ DTU Fotonik, Technical University of Denmark, DK-2800 Kgs. Lyngby, Denmark \\ ${ }^{2}$ Photonic Network System Laboratory, National Institute of Information and Communications Technology, Tokyo 184-8795, Japan \\ ${ }^{3}$ Fujikura Ltd, 1440, Mutsuzaki, Sakura, Chiba, 285-8550, Japan \\ E-mails: ${ }^{*}$ fdro@fotonik.dtu.dk, ${ }^{\dagger}$ Georg.rademacher@nict.go.jp
}

\begin{abstract}
Intra-modal four-wave mixing (FWM) and all-optical compensation by optical phase conjugation is investigated over 2-spans of 3-mode fiber with the power of the generated FWM products reduced by 5 to $20 \mathrm{~dB}$ in different scenarios. () 2020 The Author(s)
\end{abstract}

\section{Introduction}

Over the past few years, a strong research focus has been directed towards investigating strategies to increase the transmission rate of optical communication systems. Space division multiplexing (SDM) technologies, including multi-core fiber (MCF) and few-mode fiber (FMF) transmission, have been proposed as promising techniques to achieve such a goal, while potentially reducing energy consumption through components and subsystems integration. In FMFs, however, the presence of several co-propagating modes requires consideration of the impact of both intra- and inter-modal Kerr nonlinear interaction. Along this direction, a number of continuous-wave (CW) investigations of Kerr nonlinearity in FMFs have been reported, with a key focus on inter-modal four-wave mixing (FWM) [1-3] and cross-phase modulation (XPM) [4]. Additionally, a first system-level experiment has already shown that both intra- and inter-modal Kerr nonlinearity can significantly impair the signal quality [1]. For singlemode fiber (SMF) transmission, compensation of (intra-modal) Kerr nonlinearity has been investigated intensively. Optical approaches to nonlinearity compensation, such as optical phase conjugation (OPC), have the advantage of broadband operation over their digital counterparts [5]. Recently an OPC scheme implemented in a FMF has been demonstrated, and a performance improvement over transmission without OPC has been shown for SMF links [3].

In this work, we focus on the characterization and all-optical compensation of intra-modal FWM for 3-spatial mode transmission. The power of the Kerr products generated through FWM is measured under continuous-wave $(\mathrm{CW})$ operation for both $\mathrm{LP}_{01}$ and $\mathrm{LP}_{11}$ modes. One and two-span systems are considered and the measurements are compared to theoretical predictions. Additionally, OPC-based nonlinearity compensation is demonstrated, showing a significant reduction of the Kerr power for all transmission scenarios considered. This experiment shows the potential for extending optical nonlinearity compensation techniques to transmission systems based on FMFs.

\section{Experimental setup for nonlinearity characterization and compensation}

The experimental setup is shown in Fig. 1. At the transmitter side, two CW sources, one high-power fixedwavelength laser $\left(\mathrm{S} 1, \lambda_{\mathrm{S1}}=1540 \mathrm{~nm}\right)$ and a weaker swept laser $(\mathrm{S} 2)$, are used as inputs to trigger FWM in the FMF, similarly to [5]. The combined seed lasers are boosted by Erbium-doped optical amplifiers (EDFAs) followed by optical-band pass filters (OBPFs) to remove out-of-band noise and connected to the inputs of a 3D waveguide-based mode-selective multiplexer (mode mux). Two parallel transmitter paths are considered, either connecting only one path to input 1 of the mux (injecting only the $\mathrm{LP}_{01}$ mode), only to input 3 (only one of the $\mathrm{LP}_{11}$ modes), or
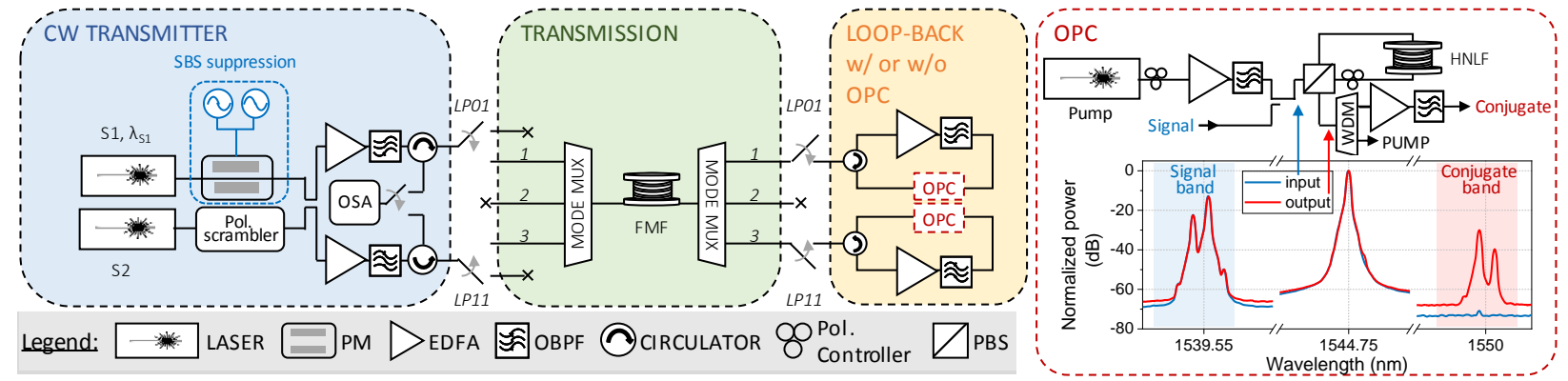

Fig. 1. Experimental setup for characterizing and compensating for Kerr nonlinearity in FMF-transmission. Right inset: OPC setup and input and output spectra for the $\mathrm{OPC}$ used for the $\mathrm{LP}_{01}$ mode (the $\mathrm{LP}_{11}$-mode path is virtually identical). 
connecting both paths to inputs 1 and 3 ( $\mathrm{LP}_{01}$ and one $\mathrm{LP}_{11}$ modes simultaneously). The launch power into the FMF is set to 13 and $14 \mathrm{dBm}$ for $\mathrm{LP}_{01}$ and $\mathrm{LP}_{11}$, respectively, to ensure the generation of a FWM idler with sufficient optical signal-to-noise ratio (OSNR) even for wide frequency separation. The 1-dB higher launch power for LP 11 is chosen to partially compensate for the additional mode mux loss and larger effective area of LP $\mathrm{P}_{11}$-modes. The transmission fiber is a 3-spatial-mode graded-index FMF [6] with the properties summarized in Fig. 2(a), including the relatively low cross-talk between the two mode groups. At the FMF output, a mode demultiplexer (demux) separates the three modes. The demux outputs can be looped back, with or without OPC, leading to two-span transmission through bi-directional propagation in the FMF. Note that the LP $\mathrm{L}_{11}$ input/output 2 of the mode mux/demux is always discarded, and as such so is half of the $\mathrm{LP}_{11}$ mode power. Finally, a high-resolution optical spectrum analyzer (OSA) is used to record the spectra after transmission (one or two looped-back spans) as the frequency separation between S1 and S2 is varied. For the FMF under test, the differential mode delay (DMD) ensures that inter-modal phase matching takes place only for frequency separations beyond $10 \mathrm{~nm}$, i.e. well outside the considered measurement range.

As S1 acts as FWM pump, it is spectrally broadened through phase modulation (PM) with two radio-frequency tones (100 and $300 \mathrm{MHz}$ ) to mitigate stimulated Brillouin scattering (SBS), which is particularly detrimental given the bi-directional propagation through the FMF. This limits the minimum frequency spacing measurable to 2 GHz. Additionally, S2 is polarization scrambled $(2.4 \mathrm{krad} / \mathrm{s})$ to average out random mode rotation, and the max-hold of the OSA is used [5]. In the loop-back configuration, chosen to ensure two identical transmission spans for OPC [7], the FMF insertion loss is compensated for with an additional EDFA. Then, in order to investigate the possibility to compensate for the nonlinear distortion accumulated during transmission, an optical phase conjugation (OPC) stage is inserted prior to signal amplification. The single-mode OPC stage relies on a standard single-pump FWM stage in a polarization-diversity loop configuration as shown in Fig.1, right inset. A high-power CW laser (OPC pump) is coupled together with the signal band into a single-mode highly nonlinear fiber (HNLF) through a polarization beam splitter (PBS) such that two orthogonal polarizations counter-propagates in the HNLF [8]. At the polarizationdiversity loop output, the OPC pump and the signal band are filtered out and only the conjugate band is looped back into the FMF. The signal and pump power into the OPC stage have been optimized to limit the OSNR degradation of the converted idlers, while avoiding additional nonlinear distortion due to signal/conjugate propagation through the HNLF (see spectra in Fig. 1) [8]. Two separate and identical OPC stages were implemented, such that up to two modes can be simultaneously (but not jointly) conjugated. Ideally, all three modes should be jointly conjugated, for example by replacing the parallel single-mode OPC stages with one multi-mode OPC stage, extending [3].

\section{Experimental results and discussion}

The experimental results are shown in Fig. 2(b-f) where the measured FWM power is reported together with the theoretical profiles [5]. The same analytical expressions have been used for $\mathrm{LP}_{01}$ and $\mathrm{LP}_{11}$ modes, by simply adjusting dispersion and effective area accordingly. Fig. 2(b) shows the FWM curves measured directly at the output of the FMF without loopback (single-span transmission). Good agreement with the theoretical prediction is shown for both modes. For a frequency separation beyond $30 \mathrm{GHz}$, the theoretical fit worsens for the $\mathrm{LP}_{11}$ mode. We believe this is the result of a stronger frequency-dependent mode rotation which is not taken into account by the analytical model and it is only partially averaged out by the polarization scrambling. Comparing $\mathrm{LP}_{01}$ and $\mathrm{LP}_{11}$, an approximate 3-dB decrease in FWM power is seen by moving to the higher-order mode. This is attributed to mode mixing and the consequent reduction in FWM [9]. Moving to the more interesting two-span scenario, Fig. 2(c,d) show the FWM power when only one mode is launched and then looped back. Compared to the single-span curves (Fig. 2(b)), the FWM power without OPC is increased by $3 \mathrm{~dB}$, following the expected scaling with the number of nonlinear regions. For the $\mathrm{LP}_{01}$ mode (Fig. 2(c)), a good agreement with the theory is shown both with and without OPC. The OPC-based compensation decreases the FWM power by almost $20 \mathrm{~dB}$ at low frequency separations. For larger frequency separations, the OSNR degradation in the OPC becomes dominant reducing correlation with the fitted line. Moving to the $\mathrm{LP}_{11}$ mode (Fig. 2(d)), the curves follow the theoretically expected trends and the OPC enables more than $10 \mathrm{~dB}$ of FWM power suppressions. The match with the theoretical fit is worse than for LP $\mathrm{P}_{01}$ as half of the modal content (output 2) is not conjugated and the frequency-dependent mode rotation is not considered by the theoretical model. As this mode-rotation can be considered an extension of frequency-dependent polarization mode dispersion (PMD), it is expected that the OPC compensation worsens with it [10]. For both LP $_{01}$ and LP 11 modes without OPC, a peak at an 11-GHz separation can be seen. That peak is the result of the amplification of S2 (and S2-S1 idler) in the second span through S1's SBS. Moving from $\mathrm{LP}_{01}$ to $\mathrm{LP}_{11}$, the peak is significantly decreased, as expected from the larger effective area of the latter [11]. Finally, Figs. 2(e,f) consider the joined propagation of both $\mathrm{LP}_{01}$ and $\mathrm{LP}_{11}$ modes through the FMF. The FWM power is shown in Fig. 2(e) and 2(f), when 

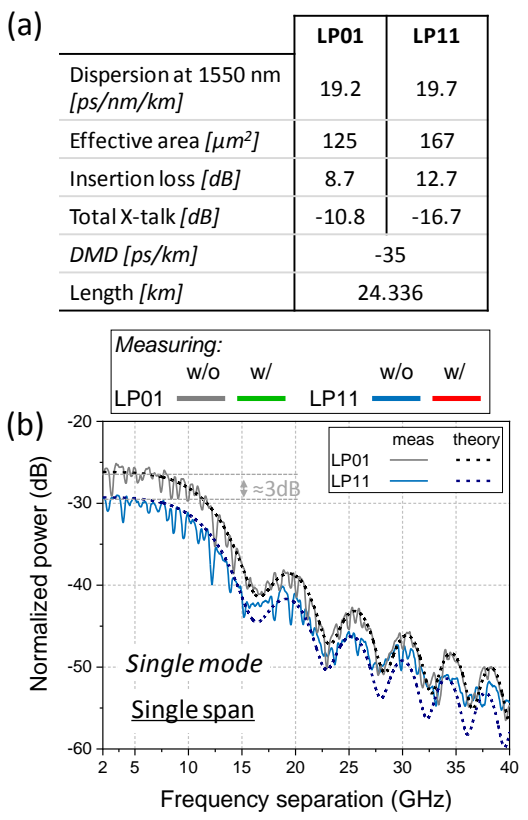
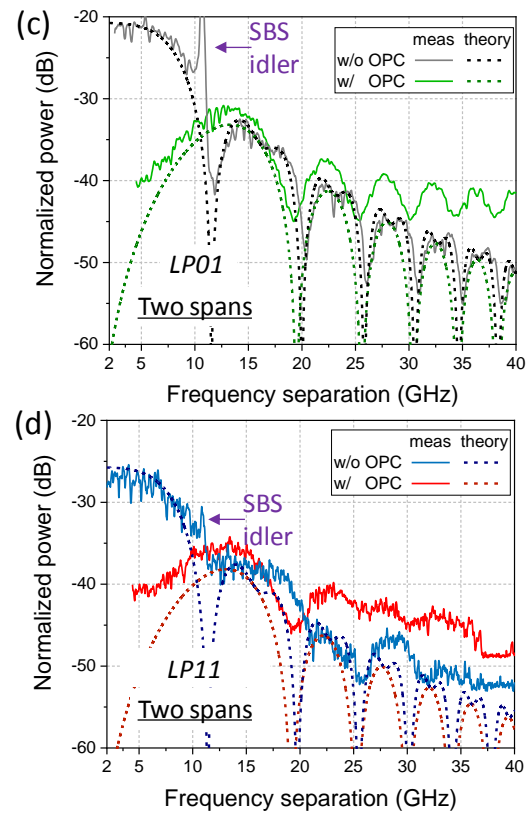
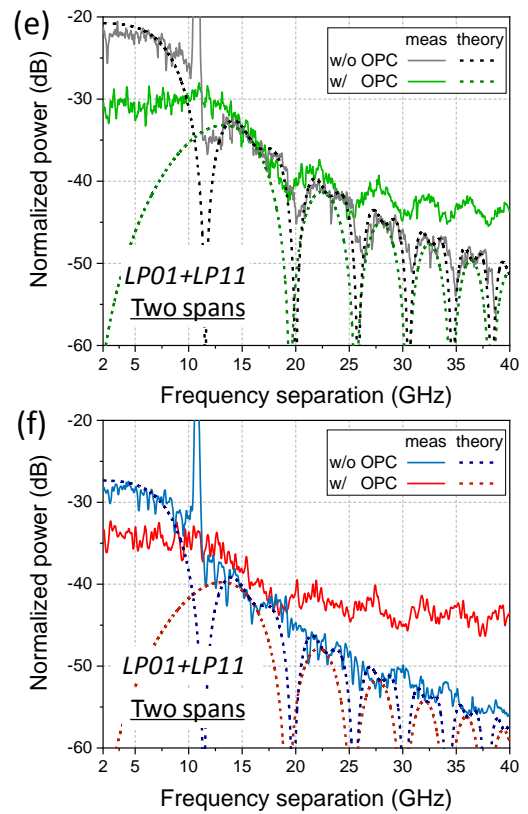

Fig. 2. (a) FMF (including mux and demux) properties, and (b-f) measured FWM power for one transmission span (b) and two transmission spans (bi-directional propagation, c-f) for $\mathrm{LP}_{01}$ alone (c), $\mathrm{LP}_{11}$ alone (d) and joint $\mathrm{LP}_{01}+\mathrm{LP}_{11}$ propagation measuring $\mathrm{LP}_{01}(\mathrm{e})$ and $\mathrm{LP} \mathrm{P}_{11}(\mathrm{f})$.

measuring the $\mathrm{LP}_{01}$ and $\mathrm{LP}_{11}$ mode, respectively. For the $\mathrm{LP}_{01}$ mode (Fig. 2(e)), a good match with the theory can still be seen for transmission without OPC. The compensation through OPC instead is impaired by the copropagating modes (coherent cross-talk) and the use of separate OPC stages for $\mathrm{LP}_{01}$ and LP 11 modes. Even though the mode coupling is relatively low between mode groups, it still has a non-negligible impact on the field symmetry required for achieving nonlinearity compensation. Nevertheless, the compensation is still in excess of $10 \mathrm{~dB}$. Finally, the measurements for the $\mathrm{LP}_{11}$ mode output are shown in Fig. 2(f). In this case, all the previously mentioned impairments, e.g. coherent modal cross-talk, lack of joined $\left(\mathrm{LP}_{01}\right.$ and $\left.\mathrm{LP}_{11}\right)$ conjugation, dropping of half of the modal (output 2), and frequency-dependent mode-rotations, further worsen the matching with the simple theory here considered. Even for this worst case, though, the benefit from the OPC is still visible and a modest FWM compensation of approx. $5 \mathrm{~dB}$ can be seen at narrow frequency separation.

\section{Conclusions}

The impact of intra-modal nonlinearity is investigated for transmission of CW signals in a 3-spatial-mode FMF. The measured FWM power for single- and two-mode transmission is in good agreement with the simple single-mode theory. Furthermore, nonlinearity compensation through OPC has been demonstrated for LP $\mathrm{P}_{01}$ and $\mathrm{LP}_{11}$ modes, showing FWM power reductions between 5 and $20 \mathrm{~dB}$ depending on the scenario considered. These results highlight the potential of mid-span OPC for nonlinearity compensation even when moving to few-mode fiber transmission.

\section{Acknowledgments}

We thank ERC CoG FRECOM (771878), DNRF Research CoE SPOC (ref. DNRF123), UMF INP project SCQC and JSPS KAKENHI (18K13761).

\section{References}

[1] G. Rademacher, et al., "Investigation of Intermodal Nonlinear Signal Distortions in Few-Mode Fiber Transmission,” J. Light. Tech. 37, 1273$79,2019$.

[2] S. Friis, et al., "Inter-modal four-wave mixing study in two-mode fiber," Opt. Expr. 24, 30338-49, 2016.

[3] I. Sackey, et al., "Fiber Nonlinearity Mitigation Using Optical Phase Conjugation Based on inter-modal FWM," ECOC 2019, p. Th.2.E.2.

[4] R.J. Essiambre et al., "Experimental observation of inter-modal crossphase modulation in few-mode fibers," IEEE Photon. Technol. Lett. 25, 535-538, 2013.

[5] M.A.Z. Al-Khateeb, et al., "Analysis of the nonlinear Kerr effects in optical transmission systems that deploy optical phase conjugation," Opt. Expr. 26, 3145-60, 2018.

[6] R. Maruyama, et al. "Two mode optical fibers with low and flattened differential modal delay suitable for WDM MIMO combined system," Opt. Expr. 22, 14311-14321, 2014.

[7] K. Solis-Trapala, T. Inoue, S. Namiki, "Nearly-ideal optical phase conjugation based nonlinear compensation system," OFC 2014 , p. W3F.8.

[8] F. Da Ros, et al., "Link-Placement Characterization of Optical Phase Conjugation for Nonlinearity Compensation," OFC 2018, p. W3E.3

[9] A. Mecozzi, et al.," Coupled Manakov equations in multimode fibers with strongly coupled groups of modes," Opt. Expr. 20, 23436-41, 2012.

[10] M.E. McCarthy, et al. "PDM tolerant nonlinear compensation using in-line phase conjugation," Opt. Expr. 24, 3385-92, 2016.

[11] N. Mathew, et al, "Improved SBS limited parametric conversion by use of few mode fibers," ECOC 2018, p. We2.1. 